\section{(6) OPEN ACCESS}

\title{
A mobile revolution for healthcare? Setting the agenda for bioethics
}

\author{
Federica Lucivero, ${ }^{1}$ Karin R Jongsma ${ }^{2}$
}

${ }^{1}$ Ethox Centre, Wellcome Centre for Ethics and Humanities, Big Data Institute, University of Oxford, Oxford, UK

${ }^{2}$ Julius Center, University Medical Center Utrecht, Utrecht University, Utrecht, The Netherlands

\section{Correspondence to} Dr Federica Lucivero, Ethox Centre, Wellcome Centre for Ethics and Humanities, Big Data Institute, University of Oxford, Oxford OX37LF, UK; federica.lucivero@ethox.ox. ac.uk

Received 31 December 2017 Revised 17 May 2018 Accepted 30 May 2018 Published Online First 15 June 2018

\section{Check for updates}

(c) Author(s) (or their employer(s)) 2018. Re-use permitted under CC BY-NC. No commercial re-use. See rights and permissions. Published by BMJ.

To cite: Lucivero $F$ Jongsma KR. J Med Ethics 2018:44:685-689.

\section{ABSTRACT}

Mobile health (mHealth) is rapidly being implemented and changing our ways of doing, understanding and organising healthcare. mHealth includes wearable devices as well as apps that track fitness, offer wellness programmes or provide tools to manage chronic conditions. According to industry and policy makers, these systems offer efficient and cost-effective solutions for disease prevention and self-management. While this development raises many ethically relevant questions, so far mHealth has received only little attention in medical ethics. This paper provides an overview of bioethical issues raised by mHealth and aims to draw scholarly attention to the ethical significance of its promises and challenges. We show that the overly positive promises of mHealth need to be nuanced and their desirability critically assessed. Finally, we offer suggestions to bioethicists to engage with this emerging trend in healthcare to develop mHealth to its best potential in a morally sound way.

\section{INTRODUCTION}

In April 2015, Apple officially launched the Apple Watch. Already before its take-off, many online fora and media published discussions on how the development of the Apple Watch confirmed the multinational company's interest to enter the health and wellness market. So it was not a surprise that on the very day the Apple Watch began shipping, already 264 Apple Watch apps related to health or fitness were found in the App Store. These apps (mobile applications) included 'fitness' (or workout) programmes, systems for medication adherence, hydration, and fertility and pregnancy tracking systems. As this example suggests, the world of wearable devices has been connected to the world of health and well-being at its very onset.

The Apple Watch is only one among many examples in the emerging market of wearable devices, apps and sensors offering health-related services, which are also described as mobile health (mHealth). ${ }^{12}$ mHealth is a broad label for a variety of services and technologies supported by mobile devices, such as smartphones, patient monitoring devices, personal digital assistants and other wireless devices to improve healthy behaviours, quality of life and well-being of individuals. An increasing number of websites showcase sleek wearable gadgets with apps continuously capturing body movements, producing graphs of daily calorie consumption or workouts, and providing health advice and self-management tools to chronic patients. Healthy individuals and chronic patients and healthcare professionals are expected targets of the mobile revolution. According to digital health proponents, like cardiologist Eric Topol or 'medical futurist' Bertalan Mesko, the healthcare of the future will be powered by digital tools ${ }^{34}$; market reports, user surveys and policy strategic documents by national and international organisations confirm that the mHealth revolution is on its way. ${ }^{12}$ According to a market report, $97000 \mathrm{mHealth}$ apps were released in 2013, and the expected revenue would be of \$26 billion in 2017, while 485 million wearable devices can be expected to be sold in $2018 .^{5}$ User uptake also seems to increase: as shown in a 2012 Pew Institute report, 31\% of cell phone owners and $52 \%$ of smartphone owners have used their phones to look up health or medical information, and $19 \%$ of smartphone owners have downloaded an app specifically to track or manage health. ${ }^{6}$ More importantly, mHealth receives support from regulatory institutions such as the European Commission, arguing that it could be one of the tools to tackle the challenges faced by European healthcare systems, such as the ageing of the population and increased budgetary pressure. ${ }^{2} \mathrm{mHealth}$, as a subset of the broader field of 'digital health' (or digital medicine), comes with promises of revolutionising healthcare by increasing patients' self-management and empowerment, fostering efficiency and disease prevention, and promoting accessibility to health around the globe. The rapid growth of this field has up until now only received little attention from bioethicists. In this paper, first we describe and critically assess the promises of mHealth and their ethical relevance. Subsequently, we offer some suggestions to promote further ethical reflection on this rapidly growing development.

\section{MHEALTH: THE PROMISE OF A REVOLUTION}

The expectation of a 'mobile revolution' in healthcare is based on the fact that mobile phones are always in our pockets, portable and increasingly cheap (eg, ref ${ }^{3}$ ). According to these narratives, the portability of mHealth systems and the ubiquity of the mobile network allow patients to freely move around while being checked remotely by healthcare providers and monitoring themselves outside the spaces traditionally dedicated to healthcare. This results in a wider range of places for healthcare and a change of roles for patients and for physicians. Mobility is considered revolutionary because it allows for people in rural areas to be as connected and cared for as people in more equipped urban 
places. ${ }^{16}$ Furthermore, although the relatively cheap and easy movement of health data from sites of collection to sites of analysis and decision-making allows for the inclusion of more datarich and real-life data in care, it also requires to rethink roles and responsibilities in the data interpretation and therapeutic decisions. As it has happened in the past in the fields of nanotechnology, genetics and personalised medicine, mHealth claims to revolutionise healthcare and solve many pressing healthcare challenges. The narrative of a revolution is based on a certain techno-optimism, which rests on the promise that technological inventions are good in themselves. ${ }^{7}$ As it has been pointed out in other cases, such claims of healthcare revolutions are rhetorical devices and they need to be assessed carefully with respect to their plausibility ${ }^{8}$ before we can engage in a reflection on the new ethical challenges that these technologies arise. At the same time, however, mHealth does indeed initiate new practices in the medical domain which need careful assessment, whether they are novel ethical challenges or more familiar ones. Earlier debates around revolutionising technologies, in fact, have shown that we have to critically assess these far-fetched promises to steer the development of technologies in such a way that we can benefit from its positive effects, but also anticipate downsides and possible risks.

In the following, we critically analyse three recurrent promises of mHealth-fostering efficiency and prevention in healthcare, increasing patient self-management and empowerment, and promoting global accessibility to health-showing their "rhetorical contradictions" : not only expectations often do not resonate with their practical use in healthcare, also they are not necessarily as positive as they are presented, but instead raise several ethical issues that need consideration.

\section{Efficiency and prevention}

mHealth is argued to offer efficient and cost-effective solutions for disease prevention, monitoring and management. Take for example an app that uses smartphone cameras to check moles for skin cancer risk (eg, https://skinvision.com/): the user holds the device over a spot on her skin and takes a picture, and the app's algorithm immediately analyses the spot and recommends an action to take (eg, whether the user should go to a specialist) or provides relevant information on skin cancer. This system allows users to archive their skin pictures, keep track of changes over time and share them with their doctor. These types of apps claim to prevent risks of skin cancer by offering a cheap tool for early self-assessment.

To live up to these promises, mHealth systems, like other medical interventions, should not harm and should (preferably) benefit users. However, despite the hype around mHealth, there are still many uncertainties around the safety, reliability and accuracy of mHealth systems. ${ }^{9}{ }^{10}$ Risks include security issues and harms that may derive from potential disclosure of sensitive information to third parties or identity theft, ${ }^{11}$ and risks of false results. For example, a skin screening app may not be accurate and might fail to recognise an early-stage melanoma, falsely reassuring rather than alerting the user. ${ }^{12}$ Other concerns have been raised concerning the efficiency of these devices: it has been argued that most sensors are unreliable in their signal detections, that it is unclear whether these devices can induce people to change their behaviour towards healthier lifestyles, ${ }^{13}$ and that clinical professionals are unaware of how to integrate these systems in their workflow. ${ }^{14}$

Moreover, when weighting the benefits and harms of mHealth, one needs to think of which benefits and harms are involved, and to consider who will actually benefit and who will experience the harms from such technologies. Some apps are primarily helpful for patients and may simultaneously help physicians, healthcare personnel or commercial parties to monitor patients, do research or sell products. The benefits and risks may not be evenly distributed among these stakeholders. It is important to ensure that the risks of harm will not predominantly lie at the user/patient side, because they are the more vulnerable actors and may have no understanding of or protection against such harms.

In general, despite the promise of providing an effective and efficient tool for disease prevention, the actual benefits of mHealth are still unclear. Although mHealth systems are sometimes presented as a replacement of traditional care, it is unlikely that they will completely replace traditional healthcare. Empirical research has indicated that patients and physicians are happy about the quality of web consultations, ${ }^{15}$ but little is known about their safety, feasibility, cost-effectiveness and efficiency. It is not clear in what contexts and situations these tools are the best possible option to meet patients' care needs: for example, it may not always be desirable, as faceto-face contact can be preferred, more efficient or simply irreplaceable, because of the need for physical examination, sensitivity of the problems, validation of mHealth data or for maintenance of the doctor-patient relationship. Also, more research is needed to understand which types of interventions and monitoring can be left to apps and wearables, and when personal, synchronous care relationship will need to be kept to ensure good care.

\section{Self-management and empowerment}

Another promise is that mHealth will empower patients. ${ }^{2}$ Many apps and wearable sensors provide self-management functions for patients or healthy citizens who want to engage in healthy lifestyles. Having access to one's own health data, without the interference of health professionals, is argued to be a prerequisite to understanding, controlling and managing one's behaviour, and therefore regarded as empowering patients and supporting their autonomy. ${ }^{16-18}$ An example is offered by the apps provided mymhealth.com for patients with asthma, chronic obstructive pulmonary disease, diabetes and heart disease that enable patients to 'achieve their goals and self-management' while being followed by a clinical team (see https://mymhealth.com/).

The claim that these digital tools empower patients and support their autonomy through self-management should be critically assessed with regard to the degree and types of autonomy that these tools promote. The underlying idea of self-management of such apps induces patients to comply to a strict (medical) regimen rather than enhancing their self-determination. ${ }^{19}$ As these devices influence or direct the behaviour of the users, concerns have been uttered that these technologies may be disciplining the users to perform specific medical tasks, rather than truly empowering them. ${ }^{17}$ Moreover, as described in the context of governmental surveillance of internet use, the so-called 'chilling effects' ${ }^{20}$ can make users of mHealth adapt their behaviours when using wearables and apps because of the feeling they are being monitored, rather than because they are themselves motivated to behave in a certain way. This means that mHealth technologies indirectly hinder them to act in the way they would have otherwise, and raise questions concerning the true meaning of the promised patient empowerment. Not only expectations that apps and self-monitoring devices empower patients are deceptive because they promote a specific medical quantified 
regimen rather than supporting a plurality of self-determined patients' value ${ }^{i}$, they also seem to assign extra responsibilities to patients. In fact, these apps and wearables delegate some tasks to patients that are traditionally carried out by healthcare professionals (eg, monitoring vital signals, updating symptoms) and continuously demand patients to perform tasks within a certain time frame. This shift of tasks is the flip side of having direct access to health data and falls into the general neoliberal trend of shifting responsibilities from the state (or healthcare providers/experts) to citizens (or individual patients), a trend often referred to as 'responsibilisation' (see http://nuffieldbioethics.org/report/personalised-healthcare-2/ what-is-personalised-healthcare). Several concerns have been raised towards such trend and are likely to apply to the field of mHealth ${ }^{18 i i}$ : first of all, it may charge patients with additional practical burdens and labour which would otherwise be taken up by healthcare professionals. ${ }^{21}$ Also, such burden may cause unduly emotional stress for patients who may feel left alone to their own care by the system. Furthermore, as several empirical studies indicate, merely having access to data does not imply that people have an increased control over their behaviour and health outcomes, as for behavioural change to happen the intervention of a health professional or counsellor is often necessary. ${ }^{22}$

Whether the expectations of empowering patients are indeed met or not, an aspect that needs close consideration is how these devices and practices influence the doctor-patient relationship. It is not only a matter of introducing more distance in the doctor-patient interaction via online tools, but also a matter of giving patients tools to control their health and rethinking the roles that doctors have in healthcare. ${ }^{23}$ By shifting tasks and responsibilities, proposing specific roles and offering tools to understand health and its correlation with lifestyle, these systems introduce new interactions between care providers and patients. This raises questions on how these relationships will take shape, whether valuable features of traditional relationships will get lost, whether medical training needs to be rethought or patient expectations redirected.

Yet another critical aspect related to promises to support patients' autonomy concerns the confidentiality and control of data. Data collected and processed by wearable sensors and apps may contain sensitive information that is made accessible to several actors, as once such data are obtained they can be reproduced and used endlessly. For example, data may be analysed by the manufacturers for improving the app performance or sold to third parties for research or marketing. Users of such wearables and apps often have limited control over who has access to their data behind the scenes. Third parties' access to health-related data can be harmful for app users who could, for example, be profiled by marketing agencies and receive targeted unwanted or upsetting advertisements, ${ }^{24}$ or incur in discriminatory policies by insurance companies or employers. Although safeguards are in place and users have the right to

\footnotetext{
${ }^{i}$ Sharon and Zandbergen ${ }^{33}$ critically assess this claim and show how, in some cases, measuring practices of 'quantified selvers' (people using selftracking tools to measure their lives) embody different values beyond the ones from the medical sciences. For example, a woman would track her symptoms after her mother's death as a way to understand and process her grief.

ii Natasha Dow Schüll ${ }^{34}$ offers a critical perspective on this point suggesting that self-tracking devices do not increase responsibilisation in users but rather are the object of a process of de-responsibilisation as users delegate to them some tasks (eg, reminding medications) that they would otherwise have to personally accomplish.
}

know what data will be collected, how these will be processed and to whom these will be distributed, this appear to be problematic and complicated in practice in the age of increasing data collection and big data analytics ${ }^{\text {iii. }}{ }^{25}$ Just to mention two issues: first, in a context wherein credit card purchases reveal individuals' lifestyle choices (eg, eating or working out habits) and enable correlations with health conditions through linkages with medical or health data (collected via apps, for example), the distinction between sensitive and non-sensitive data is ambiguous, and the definition of health-related data becomes too broad to be meaningful. ${ }^{26}$ Second, traditional safeguards in the clinical context, like practices of informed consent, are inapplicable in a consumer-focused domain where the mediation of healthcare professionals and researchers is shrinking. In an age of ambiguity between commercial and medical domain, confidentiality of health data is constantly challenged, and there is a need to discuss the moral basis of governance models that foster autonomous choice and encourage mHealth users to make an informed decision and control which data remain protected. ${ }^{\text {iv }}$

\section{Wide accessibility to health and social justice}

Finally, mHealth comes with the promise to make healthcare widely accessible. $^{2}$ mHealth is argued to have the potential to bring healthcare to areas that are difficult to reach, or to people who have mobility problems and cannot easily go to the hospital to receive care. An example can be found in a campaign raising health awareness in Bangladesh via SMS (text messages) to mobilise citizens for National Immunisation Day. ${ }^{1}$ In the context of this campaign, messages were sent to encourage parents to bring their children to get vaccinated, along with the event's date. A similar campaign involved pregnant women in remote villages who could register their mobile numbers to receive prenatal advice related to their gestation stage. With the spreading of mobile phone use in developing and rural areas, mHealth is considered a tremendous opportunity to improve healthcare in resource-poor countries in affordable ways.

The promise of increasing accessibility to healthcare meets several challenges. For example, mHealth interventions like the ones described above, using SMS (text messages), may not reach those who are most in need of care, either because they are illiterate or local languages are not supported by mobile phones, ${ }^{27}$ or because apps require mobile phones with a fast internet connection and some abilities to interact with these phones, thereby excluding certain groups (eg, low-income groups or elderly people with less dexterity with smartphones). These issues raise the question whether the people who actually are in need of better care or improved access to care are currently being served by mHealth technologies. Furthermore, it also indicates a certain hidden normativity in the ways in which services are offered and the expected users. Accessibility-related concerns also play a role in Western countries as users of some of these technologies (eg, iPhones, Fitbit) are on average younger, more educated and wealthier than average. These aspects threaten the promises of accessibility to health and raise questions whether mHealth exacerbates rather than mitigates issues of social justice

\footnotetext{
iii The new General Data Protection Regulation aims at addressing this issue at the European level, but how this will rule out in practice is still an open question.

${ }^{\text {iv }}$ Models like layered and tiered consent, as have been discussed in the context of direct-to-consumer genetic testing, could be helpful in the context of where similarly to direct-to-consumer-genetic testing data cross national boundaries with different data protection laws. ${ }^{35-37}$
} 
both at a global and local level by excluding groups from using these services.

\section{SETTING THE AGENDA FOR THE ETHICS OF MHEALTH}

The preceding sections explicate why we need to be critical towards the promises of mHealth and discuss the moral concerns that are inherent to these very promises. But if an ethical reflection on mHealth is needed and if current promises are often misleading, how can bioethicists and medical ethicists approach the topic? In the following, we will outline two suggestions for approaching the ethics of mHealth.

\section{From promises to practices}

When exploring potential implications of mHealth systems, we need to be aware that developers' websites, explanations or demos are only one side of the story describing the intended use. The way in which these technologies will actually be used in specific contexts by real people is a different matter, but both may be relevant for the ethical assessment of mHealth technologies. Literature on the history of technology and user studies show that there is often a gap between expected technology-driven scenarios and their actual realisation in specific societies. ${ }^{28}{ }^{29}$ When used in practice technologies often 'bite back', having the exact opposite effect in practice to what they were supposed to do. ${ }^{30}$ For example, although computers were often introduced in working spaces with the promise of creating a 'paperless office', they had the opposite effect as people tended to print the same or even more than usual to store and distribute documents. Mismatches between designers' expectations of users' preferences and habits, on the one hand, and actual uses of technologies are very frequent as technologies introduce new burdens, labour and responsibilities that remain invisible at a first sight. ${ }^{21} 31$ An investigation of actual uses is not always feasible (eg, the system is at an early stage of development or only used in few pilot studies, or logistical constraints prevent ethicists from engaging in such fieldwork). However, bioethicists can play a role, already during the development of devices, by uncovering the hidden normativity in the intended use and users, and by exploring possibilities for abuse and unforeseen effects. This type of early analysis can result in an alteration of the design of the device, app or service. To this end, not only the end-product but the criteria that inform different design stages should be explored and critically assessed. Bioethicists can ask questions like: is this particular system configuration going to exclude some users? Shall users be enabled to choose how they want their clinicians to monitor them through an app? These questions can then be discussed with manufacturers and prospective users to design more desirable products.

It is therefore important for bioethicists to go beyond the abstract promises and rhetoric of mHealth and situate the ethical analysis in real-world practices and specific contexts. As mHealth is often used as an 'umbrella term' to refer to a broad variety of devices with diverse functionalities, it is crucial to establish relevant moral differences among these objects and raise specific normative questions concerning the types of users, places where systems are used, the type of device and practices that develop around it.

\section{Beyond safety and effectiveness}

As the problem of securing sensitive health information and protecting individual privacy becomes crucial in the context of commercial gadgets collecting health data and the question of uploading these data on medical records is explored, most policy discussions around mHealth focus on issues of effectiveness and safety of these devices ${ }^{\mathrm{v}}$. While these topics are indeed crucial for the ethical assessment of mHealth as they refer to principles of beneficence and non-maleficence, these are not the only values and principles that are relevant from an ethical perspective. Issues of social justice, for example, should receive more attention. As discussed above, while mHealth has the potential to serve a wider range of people, it might as well exclude a large range of proportion of the population from care altogether. Questions with regard to who has access, who is excluded and whether those in need are actually served and get adequate care require careful analysis and justification, already during the development of such technologies. Some of these questions are not new as mHealth is perpetuating existing injustices of problems with new means. However, digital health, including mHealth, also brings about new ways of exploitation and commodification of data. Many mHealth services have an ambivalent status between lifestyle/well-being gadgets and health-related products, ${ }^{32}$ making it opaque how these services should be governed. Data shared on social media, online patient platforms or sold to third parties require ethical scrutiny, for privacy reasons and to protect vulnerable groups from harm and discrimination. Finally, as mentioned above while changing the ways in which healthcare is offered, mHealth affects the relationship between healthcare experts and patients, as well as people's understandings of self-care and management. The meanings of mHealth services for individuals and collectives should be explored both empirically and conceptually. Bioethicists are exceptionally placed to bring these more conceptual and empirical issues into the public debate and extend the current discourse beyond questions of effectiveness and safety of mHealth.

\section{CONCLUSIONS}

mHealth is rapidly entering institutional healthcare settings and our private spaces, and is surrounded with shiny promise of revolutionising medicine, but very little is known about their actual use and harms, which is important for the ethical assessment of such innovations. mHealth brings about new challenges and magnifies old challenges by its portability, the changing role of the patient and its market orientation. These changes have moral impact and have to be carefully scrutinised to develop suitable solutions. The visions, promises and the intended use of such technologies do provide an important basis for the ethical evaluation of such technologies, even when real-life evidence is scarce, as these often conceal hidden normativities. But to provide ethical guidance, these promises should also be compared with actual uses and practices. Because the enthusiastic promises may not all come true, and most likely, not for everyone, ethical reflection on mHealth development, promises and practices will help in providing orientation towards desirable pathways and required governance structures.

Our paper has indicated that mHealth technologies pose challenges to our ethical reflection. Our analysis has pointed out that we need to carefully reflect on the possible benefits of mHealth of empowering users, being beneficial to patients, reaching rural areas and providing affordable care infrastructure, as these promises show only one side of the coin. mHealth is a container concept featuring many different objects, with a diverse set of functions and potential uses, by a variety of users in different contexts. Such diversity is extremely important for the ethical assessment of such devices. To develop the potential

\footnotetext{
v See, for example, https://ec.europa.eu/digital-single-market/en/news/ new-eu-working-group-aims-draft-guidelines-improve-mhealth-apps-
} 
of mHealth to the fullest and implement it in an ethically sound way, ethical reflection should be involved from the early development stages of these devices. This would allow us to learn step by step from mistakes, inaccuracy, gaps between intended and actual use, and the situated use of such technologies.

Acknowledgements The authors would like to thank Nina Hallowell and Jenny Krutzinna for their helpful comments and remarks during the drafting of this paper, and the two anonymous reviewers for their insightful comments and suggestions.

Contributors FL conducted the primary research and drafted the first version of the paper, which was then discussed with KRJ, who produced the second version of the paper. The authors worked closely together during the writing and revision of any further versions of the manuscript.

Funding This study was partly funded by the European Commission Seventh Framework Programme (PIEF-GA-2013-624872). The Wellcome Centre for Ethics and Humanities and the Ethox Centre are supported by a Wellcome Centre Grant (203132/Z/16/Z)

Competing interests None declared.

Patient consent Not required.

Provenance and peer review Not commissioned; externally peer reviewed.

Open access This is an open access article distributed in accordance with the Creative Commons Attribution Non Commercial (CC BY-NC 4.0) license, which permits others to distribute, remix, adapt, build upon this work non-commercially, and license their derivative works on different terms, provided the original work is properly cited, appropriate credit is given, any changes made indicated, and the use is non-commercial. See: http://creativecommons.org/licenses/by-nc/4.0/.

\section{REFERENCES}

1 World Health Organisation (WHO). Global observatory. New horizons for health through mobile technologies. Geneva: World Health Organisation (WHO), 2011.

2 European Commission (EC). GREEN PAPER on mobile Health ("mHealth"), COM (2014) 219. Brussels: European Commission (EC), 2014

3 Topol EJ. The creative destruction of medicine : how the digital revolution will create better health care. New York: Basic Books, 2012.

4 Mesko $B$. The guide to the future of medicine: technology AND the human touch. 2014.

5 Research2guidance. Mobile Health Market Report 2013-2017. 2013.

6 Mechael PN. The case for mHealth in developing countries. Innov Technol Governance, Glob. 2009 http://www.mitpressjournals.org/doi/abs/10.1162/itgg.2009.4.1.103? journalCo de=itgg\#.VsRgVYTPz4g

7 Sparrow R. Revolutionary and familiar, inevitable and precarious: rhetorical contradictions in enthusiasm for nanotechnology. Nanoethics 2007;1:57-68.

8 Lucivero F. Ethical assessments of emerging technologies. 2016 http://link.springer. com/10.1007/978-3-319-23282-9

9 Lewis TL, Wyatt JC. mHealth and mobile medical Apps: a framework to assess risk and promote safer use. J Med Internet Res 2014;16:e210.

10 Royal Academy of Engineering. Health apps: regulation and quality control - Summary of a joint meeting held on 19 November 2014 hosted by the Academy of Medical Sciences and the Royal Academy of Engineering. 2015.

11 Graves JT, Acquisti A, Christin N. Big data and bad data: on the sensitivity of security policy to imperfect information. Univ Chicago Law Rev 2016;83.
12 Ferrero NA, Morrell DS, Burkhart CN. Skin scan: a demonstration of the need for FDA regulation of medical apps on iPhone. J Am Acad Dermatol 2013:68:515-6.

13 Tomlinson M, Rotheram-Borus MJ, Swartz L, et al. Scaling up mHealth: where is the evidence? PLoS Med 2013:10:e1001382.

14 Mehregany M, Saldivar E, Mehregany BM, et al. Opportunities and obstacles in the adoption of mHealth. mHealth 2012:7-20.

15 Tates K, Antheunis ML, Kanters S, et al. The effect of screen-to-screen versus faceto-face consultation on doctor-patient communication: an experimental study with simulated patients. J Med Internet Res 2017;19:e421.

16 Sharon T. Self-tracking for health and the quantified self: re-articulating autonomy, solidarity, and authenticity in an age of personalized healthcare. Philos Technol 2017;30:93-121.

17 Owens J, Cribb A. 'My Fitbit Thinks I Can Do Better!' do health promoting wearable technologies support personal autonomy? Philos Technol 2017;74.

18 Lupton D. The digitally engaged patient: self-monitoring and self-care in the digital health era. Social Theory \& Health 2013;11:256-70.

19 Schermer M. Telecare and self-management: opportunity to change the paradigm? J Med Ethics 2009:35:688-91.

20 Penney JW. Chilling effects: online surveillance and wikipedia use. Berkeley Tech LJ 2016:31:117.

21 Oudshoorn N. Diagnosis at a distance: the invisible work of patients and healthcare professionals in cardiac telemonitoring technology. Sociol Health IIIn 2008:30:272-88

22 Järvelä-Reijonen E, Karhunen L, Sairanen E, et al. The effects of acceptance and commitment therapy on eating behavior and diet delivered through face-to-face contact and a mobile app: a randomized controlled trial. Int I Behav Nutr Phys Act 2018;15:22.

23 Lucivero F. Lessons about so-called "Difficult" patients from the UK controversy over patient access to electronic health records. AMA J Ethics 2017:19:374-80.

24 Ebeling MFE. Healthcare and big data. Digital specters and phantom objects. US: Palgrave Macmillan, 2016.

25 Doerr M, Maguire Truong A, Bot BM, et al. Formative evaluation of participant experience with mobile eConsent in the app-mediated parkinson mpower study: a mixed methods study. JMIR Mhealth Uhealth 2017:5:e14.

26 Prainsack B. Personalized medicine: empowered patients in the 21st century? New York: NYU Press, 2017.

27 Bullen P. Operational challenges in the Cambodian mHealth revolution. J Mob Technol Med 2013;2:20-3.

28 Edgerton D. The shock of the old: technology and global history since 1900. 2011 https://books.google.co.uk/books?id=IdVGikvzlHoC

29 Marvin C. When old technologies were new : thinking about electric communication in the late nineteenth century. USA: Oxford University Press, 1988.

30 Tenner E. Why things bite back : technology and the revenge of unintended consequences. New York: Knopf, 1996.

31 Mol A. What diagnostic devices do: the case of blood sugar measurement. Theor Med Bioeth 2000;21:9-22.

32 Lucivero F, Prainsack B. The lifestylisation of healthcare? 'Consumer genomics' and mobile health as technologies for healthy lifestyle. App/ Trans/ Genom 2015;4:44-9.

33 Sharon T, Zandbergen D. From data fetishism to quantifying selves: Self-tracking practices and the other values of data. New Media Soc 2017:19:1695-709.

34 Schüll ND. Data for life: wearable technology and the design of self-care. Biosocieties 2016;11:317-33.

35 Jumelle AKL, Ispas I, Thuernmler C, et al. Ethical assessment in e-Health. e-Health Networking, Applications and Services (Healthcom). 2014 IEEE 16th International Conference, 2014; 262-8

36 Segura Anaya LH, Alsadoon A, Costadopoulos N, et al. Ethical implications of user perceptions of wearable devices. Sci Eng Ethics 2018;24:1-28.

37 Bunnik EM, Janssens AC, Schermer MH. A tiered-layered-staged model for informed consent in personal genome testing. Eur J Hum Genet 2013;21:596-601. 